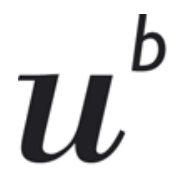

b

UNIVERSITÄT

BERN

Faculty of Business, Economics and Social Sciences

Department of Economics

The Effectiveness of Leniency Programs when Firms choose the Degree of Collusion

Winand Emons

18-16

October 2018

DISCUSSION PAPERS 


\title{
The Effectiveness of Leniency Programs when Firms choose the Degree of Collusion*
}

\author{
Winand Emons ${ }^{\dagger}$
}

Version: October 2018

\begin{abstract}
An antitrust authority deters collusion using fines and a leniency program. It chooses the probability of an investigation. Firms pick the degree of collusion: The more they collude, the higher are profits, but so is the probability of detection. Firms thus trade-off higher profits against higher expected fines. If firms are sufficiently patient, leniency is ineffective; it may even increase collusion. Increasing the probability of an investigation at low levels does not increase deterrence. Increasing the probability of an investigation at high levels reduces collusion, yet never completely.
\end{abstract}

Keywords: antitrust, cartels, deterrence, leniency.

JEL: D43, K21, K42, L40

*We thank Giancarlo Spagnolo and Igor Letina for helpful comments. The usual disclaimer applies. Authors' address: Departement Volkswirtschaftslehre, Universität Bern, Schanzeneckstrasse 1, Postfach 8573, CH-3001 Bern, Switzerland.

†Universität Bern, CEPR, Swiss Competition Commission, winand.emons@ vwi.unibe.ch. 


\section{Introduction}

A corporate leniency program reduces the sanctions for self-reporting cartel members. In 1993 the US Department of Justice revised its Corporate Leniency Program, committing itself to the lenient prosecution of the first confessor. It allows amnesty to be awarded even when an investigation has already been started. This revision is considered as the most significant policy innovation in antitrust. It substantially increased the number of detected and convicted cartels. The apparent success led the EU to adopt its own leniency program in 1996 . Other countries followed suit. ${ }^{1}$

The literature on leniency typically assumes that firms either fully collude or they do not collude at all: they set, for example, either the monopoly or the competitive price. In this paper we give up this binary choice. Our firms choose the degree of collusion - a continuous variable. They may, e.g., pick the fraction of markets on which they collude; or they may set any price between the competitive and the monopoly one. ${ }^{2}$ Firms' profits are increasing in the degree of collusion, yet so is the probability of detection: the more markets firms collude on, the higher is the probability that the antitrust authority (AA) finds out the illegal behavior once it opened an investigation. Firms thus trade-off higher profits against higher expected fines.

Legislation specifies the fine and full leniency for the first reporting firm. The fine is proportional to the degree of collusion. The AA picks the probability with which it starts an investigation. For each probability of investigation we determine the corresponding degree of collusion.

We consider two collusive strategies which differ in firms' behavior in case of an investigation. Either firms do not reveal the illegal behavior once an investigation started; they make the collusive profits, yet both firms pay the fine when detected. Or firms exploit leniency: if the AA opens an investigation, they simultaneously reveal and stop collusion during the investigation;

\footnotetext{
${ }^{1}$ See, e.g., Harrington and Chang (2009), Spagnolo (2008), or Harrington (2017).

${ }^{2}$ For example, in the Swiss construction bid-rigging cartels, firms colluded on some projects and declared others open for competition. See www.weko.admin.ch/aktuell/ 00162/index.html?lang $=$ fr....
} 
both firms then have a $50 \%$ chance of receiving leniency. Firms continue collusion after the agreed upon reporting.

First we show that if firms are sufficiently patient, leniency does not increase deterrence. Either firms collude and do not reveal in case of an investigation: then the incentive to report the cartel and get leniency is too small for patient firms. Or firms collude and reveal in case of an investigation: then firms actually collude on all markets.

Next we look at the degree of collusion as a function of the probability of an investigation. If an investigation is unlikely, firms collude and reveal in case of an investigation. Under this strategy firms collude on all markets. Increasing the probability of an investigation does not lower the degree of collusion. By contrast, if an investigation is sufficiently likely, firms collude and do not report in case of an investigation. Here the degree of collusion decreases with the probability of an investigation. Nevertheless, firms always choose a positive degree of collusion. The fine is proportional to the degree of collusion. Slightly colluding has no first-order effect on the fine, yet it raises profits.

Leniency thus produces mixed results in our set-up. With patient firms it has no bite and is, therefore, redundant. Moreover, it opens the door for the strategy collude and reveal in case of an investigation which, in turn, goes together with full collusion. Firms actually play this strategy for low probabilities of investigation. Thus, in this case leniency induces full collusion. More statements about the effectiveness of leniency are not possible without further specifying the model. Yet, our set-up generates another message. As long as the fine is proportional to the degree of collusion, firms will always collude: a small increase in the degree of collusion has no first-order effect on the expected fine but a positive first-order effect on profits.

Two of our results do not hold if firms can only choose between no and full rather than from a continuum of degrees of collusion: First, that leniency has no bite with patient firms and second, that there is always some collusion. The assessment of the efficacy of leniency thus depends on the degree of collusion firms can choose from.

Our paper builds on the analysis of leniency programs by Motta and Polo 
(2003), Spagnolo (2003), Aubert et al. (2006), and Harrington (2008). ${ }^{3}$ This literature analyzes the effects of leniency on the frequency of collusion and derives optimal fine structures.

Our basic set-up is close to Motta and Polo (2003). Besides in the degree of collusion (binary versus continuous), our framework differs from theirs in another respect: In Motta and Polo (2003) the AA chooses the probability that it opens an investigation and the probability that it successfully concludes the investigation. In our set-up the resources the AA puts into an investigation are exogenously given. Firms determine by their choice of the degree of collusion the probability that the investigation leads to a conviction. The AA, in turn, chooses the probability of an investigation.

A few papers look at variable degrees of collusion, their focus is, however, not on leniency. In Block et al. (1981) the probability of detection is an increasing function of the price; in Harrington $(2004,2005)$ it increases with the price change. In Bos et al. (2018) the probability of detection is a non-decreasing function of price. It is positive even when firms charge the competitive price and so is the fine. This means firms face a positive expected fine at the competitive price. Therefore, cartels with low overcharges do not form in the first place. ${ }^{4}$

The rest of this paper is organized as follows: The next section describes the model. In Section 3 we derive the equilibrium behavior of firms. In Section 4 we derive our results on deterrence. Section 5 concludes.

\section{Model}

Consider two potentially colluding firms. The two firms face a continuum of identical markets with mass 1 . The firms can collude on each market. More specifically, they choose the fraction $\nu \in[0,1]$ of markets on which

\footnotetext{
${ }^{3}$ Further theoretical research includes Harrington and Chang (2009), Harrington (2013), Sauvagnat (2015), and Harrington and Chang (2015); empirical and experimental research includes Bigoni et al. (2012, 2015), Brenner (2009), and Miller (2009). For a survey, see Harrington (2017).

${ }^{4}$ By contrast, Houba et al. (2010) assume like us that the probability of detection and the fine is zero at the competitive price.
} 
they collude; $\nu$ thus measures the degree of collusion. ${ }^{5}$

The legislator specifies the antitrust framework that we take as exogenously given. Within this framework the AA chooses its policy: it tries to deter collusion. At the outset the legislator announces the fine $F>0$ that a convicted firm pays whenever it colluded with the other firm on a market in the period under consideration. The legislator grants leniency to the first reporting firm. To get leniency, the reporting firm has to provide evidence of the conspiracy and it has to immediately stop the collusive conduct. If both firms choose to report, nature determines with equal probability who is first. Accordingly, in expectation each firm obtains half the leniency. We look at the case of full leniency so that the reporting firm ends up with no fine while the non-reporting firm pays $F$; if both firms report, each of them pays in expectation $F / 2$. The fine is proportional to the degree of collusion. Accordingly, if the firms collude on $\nu$ markets and are convicted, they pay a fine $\nu F$.

The AA starts an investigation with probability $\alpha \in[0,1]$. An investigation leads with probability $P$ to the detection and conviction of the cartel; we will specify $P$ as we move along.

Then an infinitely repeated game starts. The stage game in each period $t=0, \ldots$ has the following structure: Knowing $\alpha$, first firm $i, i=a, b$, decides whether it wants to communicate with the other firm or not. Firms make this decision simultaneously. If both firms choose to communicate, they create evidence that — if detected - leads to a conviction by the AA; unless both firms communicate, they do not engage in illegal behavior and there is thus no evidence thereof. ${ }^{6}$ The evidence dissolves at the end of the period. ${ }^{7}$

Next let us describe how firms collude. The two firms face a continuum of identical markets. They choose the fraction $\nu \in[0,1]$ of markets on which they collude. If the firms do not collude on a market, they compete and make profit 0 . If they collude on a market by setting the monopoly price,

\footnotetext{
${ }^{5}$ Using our notation, most of the existing literature looks at the case where $\nu \in\{0,1\}$.

${ }^{6}$ The AA thus does not make type I errors, i.e., punish non-colluding firms. See, e.g., Block and Sidak (1980) for a discussion of antitrust enforcement when courts make errors.

${ }^{7}$ Communication, even when it is not followed by anti-competitive behavior, is considered illegal.
} 
they make profit $\pi_{M}$ each. Thus, if they collude on $\nu$ markets, each firm makes profit $\nu \pi_{M}{ }^{8}$

Firms support the collusive behavior with grim-trigger strategies. If a firm deviates from collusion, Nash punishment, i.e., competition, starts and continues forever; each firm makes the static Nash profit 0. If a firm deviates while the other firm colludes, the deviating firm reaps the entire monopoly profit $2 \nu \pi_{M}$; the colluding firm's profit is $0 .^{9}$

The cartel is stable in the absence of the AA. If $\delta$ denotes the firms' common discount rate, this means that $\pi_{M} /(1-\delta)>2 \pi_{M}$, or $\delta>1 / 2$ : getting $\pi_{M}$ forever is better than getting $2 \pi_{M}$ in the first round and from then on nothing. Thus, we assume $\delta \in(1 / 2,1)$.

Then the AA starts an investigation with probability $\alpha$, leading to the investigation subgame; with probability $(1-\alpha)$ the game continues with the no-investigation subgame. In both subgames the firms choose whether they adhere to collusion or whether they deviate. Simultaneously, the firms decide whether they report any communication or not; firms make this decision knowing whether an investigation is ongoing or not.

If there is an investigation, the firms' choice of $\nu$ together with their reporting behavior determines the probability of conviction,

$$
P= \begin{cases}p(\nu), & \text { if no firm reports; } \\ 1, & \text { if firm } i \text { reports, } i=a, b, \text { or both firms report. }\end{cases}
$$

If firms do not report, the probability of conviction depends on the number of markets on which they collude. $p(\nu) \in[0,1]$ with $p(0)=0, p^{\prime}>0$ for $\nu>0$, and $p^{\prime}(1)$ finite. If firms do not collude on any market, the probability of conviction is zero. ${ }^{10}$ The more markets the firms collude on, the higher

\footnotetext{
${ }^{8}$ Alternatively, suppose the two firms face one market with demand $1-q$ where $q$ is the price. Normalizing the firms' cost to zero, the monopoly price is $q_{M}=.5$ and the monopoly profit is .25 , thus $\pi_{M}=.125$. Bertrand competition leads to $q=0$ along with zero profits. If firms collude on prices, the choice of $\nu$ corresponds to setting the price $q=.5[1-\sqrt{1-\nu}]$.

${ }^{9}$ In the Bertrand example firms fix prices: They set $q=.5[1-\sqrt{1-\nu}]$ and make profit $\nu \pi_{M}$. If a firm deviates, it slightly undercuts $q$. If firms compete, they both charge $q=0$.

${ }^{10}$ If firms decide to communicate and then pick $\nu=0$, they engage in illegal behavior that is sanctionable. Yet in our framework firms anticipate that they will choose $\nu=0$ and they will not communicate in the first place.
} 
is the probability of conviction. Furthermore, $2 p^{\prime}+\nu p^{\prime \prime}>0$ to ensure the existence of interior solutions for $\nu$ where appropriate. If one or both firms report, the evidence of collusion is unveiled and the firms get convicted for sure. $^{11}$

Legislation stipulates the fine $F$ as well as full leniency. The AA strives to deter cartels.

To summarize the the model:

- The legislator determines the fine $F$ and grants full leniency for the first reporting firm; this antitrust framework is exogenously given.

- The AA announces $\alpha$.

- Then the stage game begins:

- Firms decide whether they communicate or not.

- The AA starts an investigation with probability $\alpha$, resulting in an investigation or a no-investigation subgame.

- In both subgames firms choose whether to adhere to collusion or not and whether they report or not.

- If firms communicated and there is an investigation, they are convicted with probability $P$.

- If firms communicated and there is no investigation, they are not convicted if nobody reports. If a firm reports, they are convicted with probability 1.

Firms maximize profits with respect to their communication, price, and reporting decision. The AA chooses $\alpha$. We analyze the firms' behavior for given levels of $\alpha$.

\footnotetext{
${ }^{11}$ Firms thus possess perfect and symmetric evidence of the collusive behavior. See Blatter et al. (2018) for a set-up where firms have imperfect and asymmetric evidence.
} 


\section{Equilibrium Analysis}

Following the literature we consider two collusive strategies for firms. ${ }^{12}$ Both these strategies differ in the firms' behavior in the investigation subgame. We will, therefore, identify the two strategies with their reporting strategy in the investigation subgame:

- Firms agree to collude. In both, the no-investigation and the investigation subgame, the firms adhere to collusion and do not report. Call this strategy $N$.

- Firms agree to collude. In the no-investigation subgame they adhere to collusion and do not report. In the investigation subgame firms stop collusion and report. After an investigation with agreed upon reporting, firms continue to play the collusive strategy. Call this strategy $R .^{13}$

\subsection{Collude and not reveal}

Let us first consider strategy $N$ under which firms never report collusion. With probability $(1-\alpha)$ there is no investigation. Firms collude and make profit $\nu \pi_{M}$. With probability $\alpha$ there is an investigation. Firms collude, make profit $\nu \pi_{M}$, and face the expected fine $p(\nu) \nu F$. Next period firms continue with collusion. The expected profit per period is

$$
\pi_{N}=\nu\left[\pi_{M}-\alpha p(\nu) F\right]
$$

and the expected overall profit amounts to $\pi_{N} /(1-\delta)$.

\footnotetext{
${ }^{12}$ See, e.g., Motta and Polo (2003).

${ }^{13}$ Collude when there is no investigation, report when there is an investigation, and stop collusion after a report is clearly dominated by strategy $R$. Next consider the strategy collude when there is no investigation; when there is an investigation, firm $a$ reports while firm $b$ does not; stop collusion after a firm reported. In the investigation subgame both firms play the static Nash equilibrium leading to zero profits. If firm $b$ deviates to reporting, it doesn't change the probability of conviction, yet lowers its fine by half. Hence, it rather reports. Both firms reporting in the investigation subgame without returning to collusion thereafter is, however, dominated by strategy $R$.
} 


\subsubsection{Without leniency}

Let us first determine the firms' choice of $\nu$ absent any leniency. Maximizing (1) without any constraints yields

$$
\bar{\nu}_{N}=\left(\pi_{M} / \alpha F-p\left(\bar{\nu}_{N}\right)\right) / p^{\prime}\left(\bar{\nu}_{N}\right) .
$$

Call $\bar{\nu}_{N}$ the unconstrained choice under strategy $N . \bar{\nu}_{N}$ is increasing with $\pi_{M}$ and decreasing with $\alpha$ and $F$ : If the $\mathrm{AA}$ increases $\alpha$, the degree of collusion goes down. ${ }^{14}$ For $\alpha$ and/or $F$ sufficiently small, $\bar{\nu}_{N}>1$ so that $\nu \leq 1$ binds. More importantly, note that increasing $\nu$ at $\nu=0$ has no first-order effect on the expected fine while profits increase. Therefore, firms always choose a positive degree of collusion, i.e., $\bar{\nu}_{N}>0$.

Next let us take the firms' incentive constraint without leniency into account: In the investigation as well as in the no-investigation subgame a firm must prefer to adhere to collusion rather than deviate for a onetime increase in profit.

Suppose the AA has started an investigation so that we are in the investigation subgame. If firms continue to play $N$, their profit is $\nu\left[\pi_{M}-p(\nu) F+\right.$ $\left.\delta\left(\pi_{M}-\alpha p(\nu) F\right) /(1-\delta)\right]$. If a firm deviates from collusion, it makes profit $2 \nu \pi_{M}-p(\nu) \nu F$ : It gets the deviation profit minus the expected fine; however, from next period on the firms compete and make no profits.

Suppose we are in the no-investigation subgame. If firms continue to play $N$, their profit is $\nu\left[\pi_{M}+\delta\left(\pi_{M}-\alpha p(\nu) F\right) /(1-\delta)\right]$. If a firm deviates from collusion, it makes profit $2 \nu \pi_{M}$. Firms play $N$ rather than report and deviate in both, the investigation and the no-investigation subgame, if

$$
\nu\left[\pi_{M}+\delta\left(\pi_{M}-\alpha p(\nu) F\right) /(1-\delta)\right] \geq 2 \nu \pi_{M} .
$$

To support $N$ without leniency, firms choose the degree of collusion that satisfies the equality in (3),

$$
\tilde{\nu}_{N}=p^{-1}\left((2 \delta-1) \pi_{M} / \delta \alpha F\right) .
$$

\footnotetext{
${ }^{14}$ For example, formally we have $\partial \bar{\nu}_{N} / \partial \alpha=-\pi_{M} / \alpha^{2} F\left(2 p^{\prime}\left(\bar{\nu}_{N}\right)+\bar{\nu}_{N} p^{\prime \prime}\left(\bar{\nu}_{N}\right)\right)<0$.
} 


\subsubsection{With leniency}

Consider now the situation with leniency. Suppose the AA has started an investigation so that we are in the investigation subgame. If firms continue to play $N$, their profit is $\nu\left[\pi_{M}-p(\nu) F+\delta\left(\pi_{M}-\alpha p(\nu) F\right) /(1-\delta)\right]$. If a firm reports and deviates from collusion, it makes profit $2 \nu \pi_{M}$ : It gets the deviation profit and pays no fine; however, from next period on the firms compete and make no profits. Firms play $N$ rather than report and deviate if

$$
\nu\left[\pi_{M}-p(\nu) F+\delta\left(\pi_{M}-\alpha p(\nu) F\right) /(1-\delta)\right] \geq 2 \nu \pi_{M} .
$$

To support $N$ in the presence of leniency firms choose the degree of collusion that satisfies the equality in (5). We have

$$
\hat{\nu}_{N}=p^{-1}\left((2 \delta-1) \pi_{M} /(1-\delta+\delta \alpha) F\right) .
$$

Call $\hat{\nu}_{N}$ the constrained choice under strategy $N$. $\hat{\nu}_{N}$ is increasing in $\pi_{M}$ and decreasing in $\alpha$ and $F$. First note that, not surprisingly, $\hat{\nu}_{N}<\tilde{\nu}_{N}$. The introduction of leniency makes the deviation to not collude more attractive: by not collude and report the firm gets the deviation profit and avoids the

fine $F$. Therefore, under strategy $N$ firms choose either the unconstrained $\bar{\nu}_{N}$ or the constrained degree of collusion $\hat{\nu}_{N}$. Clearly, they do better with $\bar{\nu}_{N}$ than $\hat{\nu}_{N}$. Consequently, they will choose $\hat{\nu}_{N}$ only when it is smaller than $\bar{\nu}_{N}$. Taking into account that $\nu \in[0,1]$, we have

$$
\nu_{N}^{*}=\min \left\{\bar{\nu}_{N}, \hat{\nu}_{N}, 1\right\} .
$$

Let us now state our first result. If firms are sufficiently patient, under strategy $N$ the incentive to deviate plays no role: it is not attractive to give up the future profits from collusion for a onetime profit increase. By contrast, if they are sufficiently impatient, the incentive to deviate alone determines the degree of collusion.

\section{Lemma 1:}

a) For $\delta$ close to $1, \bar{\nu}_{N}<\hat{\nu}_{N} \forall \alpha>0$;

b) for $\delta$ close to $1 / 2, \hat{\nu}_{N}<\bar{\nu}_{N} \forall \alpha>0$. 
Proof: a) Rewrite (2) as $p\left(\bar{\nu}_{N}\right)=\pi_{M} / \alpha F-p^{\prime}\left(\bar{\nu}_{N}\right) \bar{\nu}_{N}$ and (6) as $p\left(\hat{\nu}_{N}\right)=$ $(2 \delta-1) \pi_{M} /(1-\delta+\delta \alpha) F \cdot p\left(\hat{\nu}_{N}\right)$ is increasing in $\delta$ and $\lim _{\delta \uparrow 1} p\left(\hat{\nu}_{N}\right)=\pi_{M} / \alpha F$. For $\delta$ close to one, $p\left(\hat{\nu}_{N}\right)>p\left(\bar{\nu}_{N}\right)$, because $\bar{\nu}_{N}>0$. Since $p^{\prime}>0, \hat{\nu}_{N}>\bar{\nu}_{N}$.

b) $\lim _{\delta \downarrow 1 / 2} p\left(\hat{\nu}_{N}\right)=0$, hence $\hat{\nu}_{N} \rightarrow 0$ for $\delta$ approaching $1 / 2$. Since $\bar{\nu}_{N}>0$, we have $\hat{\nu}_{N}<\bar{\nu}_{N}$ for $\delta$ close to $1 / 2$.

Note that part a) of Lemma 1 does not hold if we restrict $\nu \in\{0,1\}$. Obviously, under this restriction the interior solution $\bar{\nu}_{N}$ does not exist. Let $\alpha$ and $F$ be, for example, such that for $\nu=1$ the equality in (3) is satisfied, i.e., without leniency firms collude. Introducing leniency makes the deviation from collusion more attractive so that (5) is not satisfied for $\nu=1$. In this case leniency thus increases deterrence for all $\delta \in(1 / 2,1)$.

\subsection{Collude and reveal}

Let us now turn to the strategy $R$. With probability $(1-\alpha)$ there is no investigation. Firms collude and make profit $\nu \pi_{M}$. With probability $\alpha$ there is an investigation. Firms report, stop collusion in this period by playing the static Nash equilibrium, make 0 profit, and pay the fine $\nu F / 2$. Next period firms return to collusion. The expected profit per period is

$$
\pi_{R}=\nu\left[(1-\alpha) \pi_{M}-\alpha F / 2\right]
$$

and the expected overall profit $\pi_{R} /(1-\delta)$.

Now let us consider the incentive to deviate. In the investigation subgame both firms report. A firm does not unilaterally deviate to not report because the other firm reports: the fine it pays increases from $F / 2$ to $F$ without any benefits whatsoever.

In the no-investigation subgame if firms play $R$, they collude and do not report; their profit is $\nu\left[\pi_{M}+\delta\left((1-\alpha) \pi_{M}-\alpha F / 2\right) /(1-\delta)\right]$. If a firm reports and deviates from collusion, it makes profit $2 \nu \pi_{M}$ : It gets the deviation profit and pays no fine; however, from next period on the firms compete and make no profits. Firms play $R$ rather than report and deviate if

$$
\nu\left[\pi_{M}+\delta\left((1-\alpha) \pi_{M}-\alpha F / 2\right) /(1-\delta)\right] \geq 2 \nu \pi_{M} .
$$


Hence,

$$
\nu_{R}= \begin{cases}0, & \alpha>\pi_{M}(2 \delta-1) / \delta\left(F / 2+\pi_{M}\right) \\ 1, & \text { otherwise }\end{cases}
$$

$\pi_{R}$ is linear in $\nu$. Therefore, the optimal degree of collusion is either zero or one.

\section{Deterrence}

Before we analyze when firms actually play $N$ or $R$ under leniency, we may already state the following result: If firms are sufficiently patient, the introduction of leniency does not decrease collusion.

Proposition 1: For $\delta$ close to one, leniency does not increase deterrence.

Proof: We know from Lemma 1 that $\bar{\nu}_{N}<\hat{\nu}_{N}$ for $\delta$ close to 1 . If $\bar{\nu}_{N}<1$, firms choose the interior degree of collusion $\bar{\nu}_{N}$. If $\bar{\nu}_{N} \geq 1$, firms collude on all markets. In both cases the introduction of leniency does not change their behavior as to strategy $N$.

Leniency introduces the option to play strategy $R$. Under strategy $N$ firms always make positive profits. Therefore, if firms prefer $R$ to $N$, they must fully collude on all markets. More than full collusion is not possible under strategy $N$. Thus, collusion does not go down if firms switch from $N$ to $R$.

We know from Lemma 1 that leniency has no bite as to strategy $N$ if firms are sufficiently patient; the degree of collusion is the same as without leniency. Leniency opens the door for strategy $R$. If firms actually choose $R$, then they fully collude. Therefore, if firms continue to play $N$, the introduction of leniency does not change the degree of collusion. If firms switch to $R$, they fully collude after the introduction of leniency. Collusion goes up if $\bar{\nu}_{N}<1$; otherwise, it remains unchanged.

Proposition 1 implies that with sufficiently patient firms the introduction of leniency is not a good idea. Given firms play collude and not reveal, the option of getting leniency does not induce them to blow the whistle. 
Furthermore, leniency introduces the possibility to play collude and reveal. If firms opt for this strategy, they collude on all markets.

Let us now determine the firms' strategy choice as a function of $\alpha$.

\section{Proposition 2:}

a) For $\alpha$ small and $F \geq \pi_{M} /(p(1)-1 / 2)$, firms play $R$ and $\nu^{*}=\nu_{R}=1$;

b) for $\alpha>\pi_{M}(2 \delta-1) / \delta\left(F / 2+\pi_{M}\right)$, firms play $N$ and $\nu^{*}=\nu_{N}^{*}>0$.

Proof: a) If $\alpha$ small, $\bar{\nu}_{N}=1$ and $\pi_{N}(1)=\pi_{M}-\alpha p(1) F . \quad \hat{\nu}_{N} \leq \bar{\nu}_{N}$ and $\pi_{N}\left(\hat{\nu}_{N}\right) \leq \pi_{N}\left(\bar{\nu}_{N}\right)$ because $\bar{\nu}_{N}$ is the unconstrained choice. For $\alpha$ small, $\nu_{R}=$ 1 and $\pi_{R}(1)=(1-\alpha) \pi_{M}-\alpha F / 2 . \pi_{R}(1) \geq \pi_{N}(1)$ if $F \geq \pi_{M} /(p(1)-1 / 2)$.

b) For $\alpha>\pi_{M}(2 \delta-1) / \delta\left(F / 2+\pi_{M}\right), \nu_{R}=0$ and $\pi_{R}(0)=0 . \nu_{N}^{*}>0 \forall \alpha$, hence $\pi_{R}\left(\nu_{N}^{*}\right)>0$.

If $\alpha$ is small, the firms' unconstrained choice under strategy $N$ is $\bar{\nu}_{N}=1$ and $\pi_{N}(1)=\pi_{M}-\alpha p(1) F$. Taking the incentive to deviate into account yields $\hat{\nu}_{N} \leq \bar{\nu}_{N}$. The profit under the constrained choice cannot exceed the profit under the unconstrained choice, thus $\pi_{N}(1)$ is the upper bound for profits under strategy $N$. Under strategy $R$ firms pick $\nu_{R}=1$ for $\alpha$ small, leading to profit $\pi_{R}(1)=(1-\alpha) \pi_{M}-\alpha F / 2$. $R$ yields higher profits than $N$ if $F \geq \pi_{M} /(p(1)-1 / 2)$. Under $R$ a firm gives up $\pi_{M}$ and pays $F / 2$ in the investigation subgame; under $N$ it pays $p(1) F$ in the investigation subgame. Given $p(1)>1 / 2$, for $F$ sufficiently large firms prefer $R$.

For $\alpha>\pi_{M}(2 \delta-1) / \delta\left(F / 2+\pi_{M}\right)$, firms do not collude under $R$ which, in turn, implies zero profits. Under strategy $N$ there is always collusion leading to positive profits. From Lemma 1 we know that for $\delta$ close to one, firms choose the unconstrained $\bar{\nu}_{N}$; leniency has thus no bite. By contrast, for $\delta$ close to $1 / 2$, firms pick $\hat{\nu}_{N}$ : the degree of collusion is constrained by leniency.

For all other cases the firms' choices cannot be determined without specifying the detection probability $p(\nu)$. Firms may play strategy $N$ with the unconstrained $\bar{\nu}_{N}$ or the constrained choice $\hat{\nu}_{N}$, both of which are positive for all $\alpha$; or firms play strategy $R$ with $\nu_{R}=1$. Yet, despite this vagueness, we can make the following statement: Whatever detection probability $\alpha$ the 
AA chooses, firms will answer with a positive degree of collusion; complete deterrence is not possible in our set-up.

Proposition 2 has the following policy messages. In case a) slightly increasing $\alpha$ does not lower the degree of collusion: firms continue to collude on all markets playing $R$. However, it increases cartel desistence, i.e., firms interrupt their collusion more often and the AA collects the fine more often. In case b) increasing $\alpha$ lowers the degree of collusion (though never to zero). Whether the AA collects more fines is, however, unclear: $\alpha$ goes up, $\nu_{N}^{*}$ goes down, and so does the probability of detection $p\left(\nu_{N}^{*}\right)$ and the fine $\nu_{N}^{*} F$.

As to the introduction of leniency, Proposition 2 implies the following. For small values of $\alpha$ introducing leniency is not a good idea: firms may switch from collude and not reveal with $\nu_{N}^{*}<1$ to collude and reveal with $\nu_{R}^{*}=1$, i.e., collusion goes up. For large values of $\alpha$ firms choose collude and not reveal. Suppose without leniency firms do not choose the unconstrained degree of collusion, i.e., $\tilde{\nu}_{N}<\bar{\nu}_{N}$ with $\tilde{\nu}_{N}$ given by (4). Then leniency reduces collusion because it reinforces the incentive to deviate $\left(\hat{\nu}_{N}<\tilde{\nu}_{N}\right)$. Nevertheless, with and without leniency firms may pick the unconstrained $\bar{\nu}_{N}$, so that leniency has no effect on collusion.

The result that $\nu_{N}^{*}>0$ is of course driven by our assumption that the fine $\nu F$ is proportional to the degree of collusion. We are not aware of any legal system where this is not the case in one form or the other. For example, in Switzerland the fine is a percentage (up to 10\%) of the revenue made in Switzerland in the last 3 years. The actual percentage is adjusted for the severity of collusion (horizontal price-fixing is most severe offense), whether the firms cooperated during the investigation etc. The revenue should be positively correlated with the degree of collusion, thus so is the fine. Accordingly, our assumption that the fine goes up with the degree of collusion seems a reasonable approximation for Swiss institutions. Note that even with a constant fine the result continues to hold if $p^{\prime}(0)=0$ which is, e.g., the case for $p(\nu)=\nu^{\beta}, \beta>1$. Obviously, the result $\nu_{N}^{*}>0$ is not true if we restrict $\nu \in\{0,1\}$.

In our framework the AA chooses the probability of an investigation $\alpha$. Suppose, for example, to investigate an industry the AA needs a certain 
amount of manpower. By choosing the overall size of its staff, the AA determines the fraction $\alpha$ of industries it can investigate. Each industry is equally likely to be investigated, independently of its degree of collusion $\nu ; \nu$ only plays a role for the probability of conviction $p$. It seems conceivable that $\alpha$ is also an increasing function of $\nu$ : the more firms collude, the more suspicious the industry, the more likely it is to be investigated. While such a set-up is certainly of interest, it raises a couple of problems: Is $\nu$ more important for $\alpha$ or for $p$ ? How does $\alpha$ depend on the efforts by the AA and the degree of collusion $\nu ?^{15}$ We have avoided these modeling issues by assuming separability: the AA picks $\alpha$ while the firms strategically determines $p$.

\section{Conclusions}

In most of the existing literature on leniency firms have to decide between colluding and not colluding, a binary choice. Yet, often firms have more than two options as to collusion: they can, e.g., collude on some markets and compete on the others or the can set any price between the competitive and the monopoly one. The purpose of this paper is to study leniency programs when firms choose the degree of collusion, a continuous variable. It turns out that this affects the effectiveness of leniency programs. To asses leniency it seems thus a good idea to keep the firms' collusion possibilities in mind.

As to the introduction of leniency, our results are mixed. If the probability of an investigation is small or if firms are sufficiently patient, leniency is ineffective and may even increase collusion. In all other cases leniency may or may not reduce collusion. Firms, choose, however, always a positive degree of collusion. Complete deterrence is not possible in our framework.

\footnotetext{
${ }^{15}$ Abusing notation we could, e.g., use a contest function to define the probability of an investigation as $\alpha /(\alpha+\nu)$. With the same approach we could define $p$.
} 


\section{References}

Aubert, C., Kovacic, W., And P. Rey, 2006. The Impact of Leniency Programs on Cartels, International Journal of Industrial Organization 6, 1241-1266.

Bigoni, M., Fridolfsson, S., Le Coq, C., And G. Spagnolo, 2012. Fines, Leniency, and Rewards in Antitrust, Rand Journal of Economics 43, 368-390.

Bigoni, M., Fridolfsson, S., Le Coq, C., and G. Spagnolo, 2015. Trust, Leniency, and Deterrence, Journal of Law, Economics, and Organization, 31, 663689.

Blatter, M., Emons, W., And S. Sticher, 2018. Optimal Leniency Programs when Firms Have Cumulative and Asymmetric Evidence, Review of Industrial Organization, 52, 403-427.

Block, M. And J. Sidak, 1980. The Cost of Antitrust Deterrence: Why Not Hang a Price Fixer Now and Then?, Georgetown Law Journal 68, 1131-1139.

Block, M., Nold, F. And J. Sidak, 1981. The Deterrent Effect of Antitrust Enforcement, Journal of Political Economy 89, 429-445.

Bos, I., Davies, S., Harrington, J., And P. Ormosi, 2018. Does Enforcement deter Cartels? A Tale of two Tails, International Journal of Industrial Organization 59, 372-405.

Brenner, S., 2009. An Empirical Study of the European Corporate Leniency Program, International Journal of Industrial Organization 27, 639-645.

Harrington, J., 2004. Cartel Pricing Dynamics in the Presence of an Antitrust Authority, Rand Journal of Economics 35, 651-673.

Harrington, J., 2005. Optimal Cartel Pricing in the Presence of an Antitrust Authority, International Economic Review 46, 145-169.

Harrington, J., 2008. Optimal Corporate Leniency Programs, Journal of Industrial Economics 56, 215-246.

Harrington, J., 2013. Corporate Leniency Programs when Firms have Private Information: The Push of Prosecution and the Pull of Pre-Emption, Journal of Industrial Economics 61, 1-27.

Harrington, J., 2017. The Theory of Collusion and Competition Policy, MIT Press, Cambridge, Massachusetts, USA.

Harrington, J. And M. Chang, 2009. Modeling the Birth and Death of Cartels with an Application to Evaluating Competition Policy, Journal of the European 
Economic Association 7, 1400-1435.

Harrington, J. And M. Chang, 2015. When Can We Expect a Corporate Leniency Program to Result in Fewer Cartels?, Journal of Law and Economics 58, 417-449.

Houba, H., Motchenkova, E., And Q. Wen, 2010. Antitrust Enforcement with Price-dependent Fines and Detection Probabilities, Economics Bulletin 30, 2017-2017.

Miller, N., 2009. Strategic Leniency and Cartel Enforcement, American Economic Review 99, 750-768.

Motta, M. And M. Polo, 2003. Leniency Programs and Cartel Prosecution, International Journal of Industrial Organization 21, 347-379.

Sauvagnat, J., 2015. Prosecution and Leniency Programs: The Role of Bluffing in Opening Investigations, Journal of Industrial Economics 63, 313-338.

Spagnolo, G., 2003. Divide et Impera: Optimal Deterrence Mechanisms Against Cartels and Organized Crime, sites.google.com/site/giancarlospagnoloshomepage/ research-2/working-papers.

Spagnolo, G., 2008. Leniency and Whistleblowers in Antitrust, in Paolo Buccirossi (ed.), Handbook of Antitrust Economics, MIT Press, Cambridge, Massachusetts, USA, 259-303. 